SECTION 3. Nanotechnology, Physics.

Loshchilov Sergey Andreevich assistant, Nizhny Novgorod State Technical University

Maslennikov Dmitry Alexandrovich assistant, candidate of math and physics, Nizhny Novgorod State Technical University,

Kataeva Liliya Yuryevna dr. of math and physics, docent, professor, Nizhny Novgorod State Technical University,

\title{
ITERATIVE ALGORITHM OF THERMOKINETIC PARAMETERS ESTIMATION FOR WOOD PYROLYSIS PROCESS BY EXPERIMENTAL THERMOGRAVIMETRICAL DATA
}

An iterative algorithm of thermokinetic constants estimation was developed for researched pyrolysis process, considering the selected mathematical model of pyrolysis, by experimental data obtained in scope of thermogravimetic experiment. Estimation of thermogravimetric constants was completed for several experiments, by application of the algorithm and manual selection of temperature range for calculations. The theoretical graph of mass loss speed was plotted, considering the calculated preexponential factor and activation energy and selected pyrolysis model. Conclusion about suitability of selected mathematical model of pyrolisys and thermokinetic parameters estimation method was done, by comparison of theoretical and experimental plot of mass loss.

Keywords: pyrolysis, activation energy, preexponential factor.

\section{ИТЕРАЦИОННЫЙ АЛГОРИТМ ПОДБОРА ТЕРМОКИНЕТИЧЕСКИХ ПОСТОЯННЫХ ПРОЦЕССА ПИРОЛИЗА ДРЕВЕСИНЫ ПО ЭКСПЕРИМЕНТАЛЬНЫМ ТЕРМОГРАВИМЕТРИЧЕСКИМ ДАННЫМ}

Разработан алгоритм подбора термокинетических постоянных для исследуемого процесса, с учетом выбранной математической модели пиролиза, по экспериментальным данным, полученным в ходе термогравиметрического эксперимента. Выполнен расчет термокинетических постоянных для нескольких экспериментов, путем 
применения алгоритма поиска постоянных и ручного подбора гранич температур прочесса. Произведено построение теоретической кривой скорости потери массы с учетом вычисленных энергии активации и предэкспоненциального множителя, $c$ применением выбранной математической модели пиролиза. Сделаны выводы об адекватности выбранного метода расчета постоянных и выбранной модели проиесса пиролиза, путем сравнения экспериментальных и расчетных графиков скорости потери массы.

Ключевые слова: пиролиз, энергия активаџии, предэкспоненцииальный множитель.

\section{1. Предварительная обработка данных}

Особенностью входных данных, при термогравиметрическом эксперименте, является изменение массы не каждый момент, представленный в таблице, ввиду ограниченной точности оцифровки сигнала с гравиметрической установки. То есть имеют место группы подряд идущих отсчетов с одинаковыми показаниями массы. Поэтому производится очистка данных. В таблице каждая группа последовательных строк с постоянной массой заменяется одной строкой, а время и температура считаются как среднее между максимумом и минимумов в этой группе. Это даѐт возможность получать адекватные значения производной от массы.

Затем производится кубическая сплайн интерполяция [1], с помощью которой рассчитываются результаты вычисления температуры и массы в моменты времени $t=\left(i / N_{\text {steps }}\right) t_{\max }$, где $0 \leq i \leq$ Nsteps .

Кубическая сплайн-интерполяция - это быстрый, эффективный и устойчивый способ интерполяции функций. Основная идея метода состоит в разбиении интервала интерполяции на отрезки, находящиеся между каждыми двумя соседними узлами для получения функции в виде полинома третьей степени. На границах отрезков выполняются условия непрерывности функции, и еѐ первых двух производных. Данный метод достаточно эффективен, если интерполируемая функция достаточно гладкая. Благодаря этому обеспечивается хорошая точность в определении значений массы в промежуточных точках, что позволяет получить интерполяционную оценку экспериментальных данных на априори заданной сетке. Для обеспечения корректности сплайн-интерполяции выбор временной сетки связан с фактической продолжительностью эксперимента.

Моментам времени $t_{1}, t_{2}, \ldots, t_{n}$ и соответствуют значения интерполируемой величины $y_{1}, y_{2}, \ldots, y_{n}$. На каждом из отрезков $\left[x_{i}, x_{i+1}\right]$, $i=1,2, \ldots, n-1$ функцию приближаем при помощи полинома третьей степени:

$$
S(x)=y_{i}+c_{1 i}\left(x-x_{i}\right)+c_{2 i}\left(x-x_{i}\right)^{2}+c_{3 i}\left(x-x_{i}\right)^{3},
$$




$$
x_{i}<x<x_{i+1} .
$$

Для вычисления коэффициентов $c_{1 i}, c_{2 i}, c_{3 i}, i=1,2, \ldots, n-1$ решается система линейных алгебраических уравнений, полученная из условия непрерывности первой производной $S^{\prime}(x)$ в узлах сетки и дополнительных краевых условий на вторую производную, имеющие вид:

После сплайн-интерполяции данные о массе и температурах определяются в точках $t=\left(i / N_{\text {steps }}\right) t_{\max }$.

Относительная убыль массы вычисляется с помощью следующего соотношения:

$$
M_{i}\left(\frac{t_{j}+t_{j+1}}{2}\right) \sim \frac{\left(m_{i}\left(t_{j+1}\right)-m_{i}\left(t_{j}\right)\right)}{\left[\frac{m_{i}\left(t_{j+1}\right)+m_{i}\left(t_{j}\right)}{2}-\alpha_{i} m_{i}\left(t_{0}\right)\right]\left(t_{j+1}-t_{j}\right)},
$$

где $M_{i}$ - относительная убыль массы $i$-го компонента, $t_{i}$,- время на соответствующем $j$-м шаге сетки $\left(t_{j+1}\right), t_{j+1}-t_{j}=\Delta h t_{0}$ - начальный момент времени в анализе данной стадии, $\alpha_{i}$ - величина условно нереагирующего остатка после $i$-й стадии. Прирост массы рассчитывается в серединах отрезков в целях повышения точности.

\section{2. Вычисление термокинетических постоянных}

Основным блоком алгоритма является нахождение энергии активации и предэкспоненциалного множителя на основе экспериментальным данным по относительной убыли массы $[2$, с.3]. Предполагается, что в каждый момент времени имеет место только одна стадия пиролиза, то есть стадии не пересекаются во времени, и значения термокинетечиских постоянных не меняются на протяжении всего временного интервала принадлежащего одной стадии [3, с. 2]. Данный алгоритм принимает на вход данные об относительной убыли массы, данные о температурах в соответствующие моменты времени и отрезок температур, на котором анализируются данные.

Алгоритм вычисления энергии активации основан на модифицированном методе дихотомии. Вначале делается предположение о верхней и нижней оценке величины энергии активации $\left(E_{\min }\right.$ и $\left.E_{\max }\right)$, затем вычисляется среднегеометрическое $E_{t e s t}$. На следующем этапе выполняется оценка энергии активации как средне геометрическое.

\section{3. Решение прямой задачи химической кинетики}

Для оценки качества решения обратной задачи, для полученных параметров выполняется решение прямой задачи согласно системе уравнений (2), затем производится оценка массы твѐрдой фазы, которая и сравнивается с экспериментальными данными.

\section{4. Определение термокинетических постоянных при обработке экспериментальных данных окислительной среды}

Были обработаны предоставленные экспериментальные данные. На рис. 1, 2 представлены полученные результаты. Графики слева показывают 
зависимость производную массы относительно температуры, а правые графики показывают относительную убыль массы. График синей линией отражает экспериментальные данные, а красная линия отображает решение прямой кинетической задачи, с подобранными термокинетическими постоянными. На всех графиках расчѐтные линии более гладкие, так как не содержат погрешности эксперимента, характерную для экспериментальных данных.
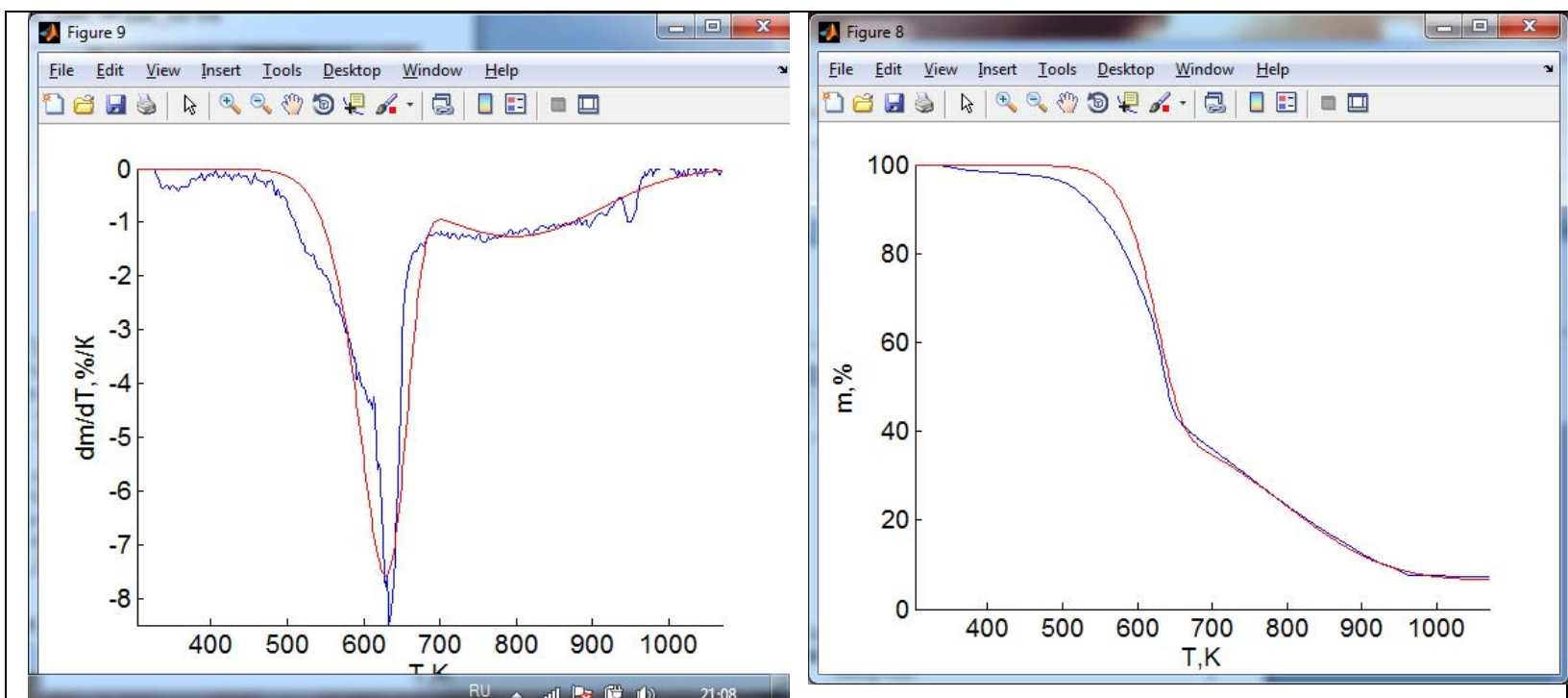

Рисунок. 1 - Экспериментальные данные и подбор термокинетических постоянных для экспериментальных данных с 5\% кислорода
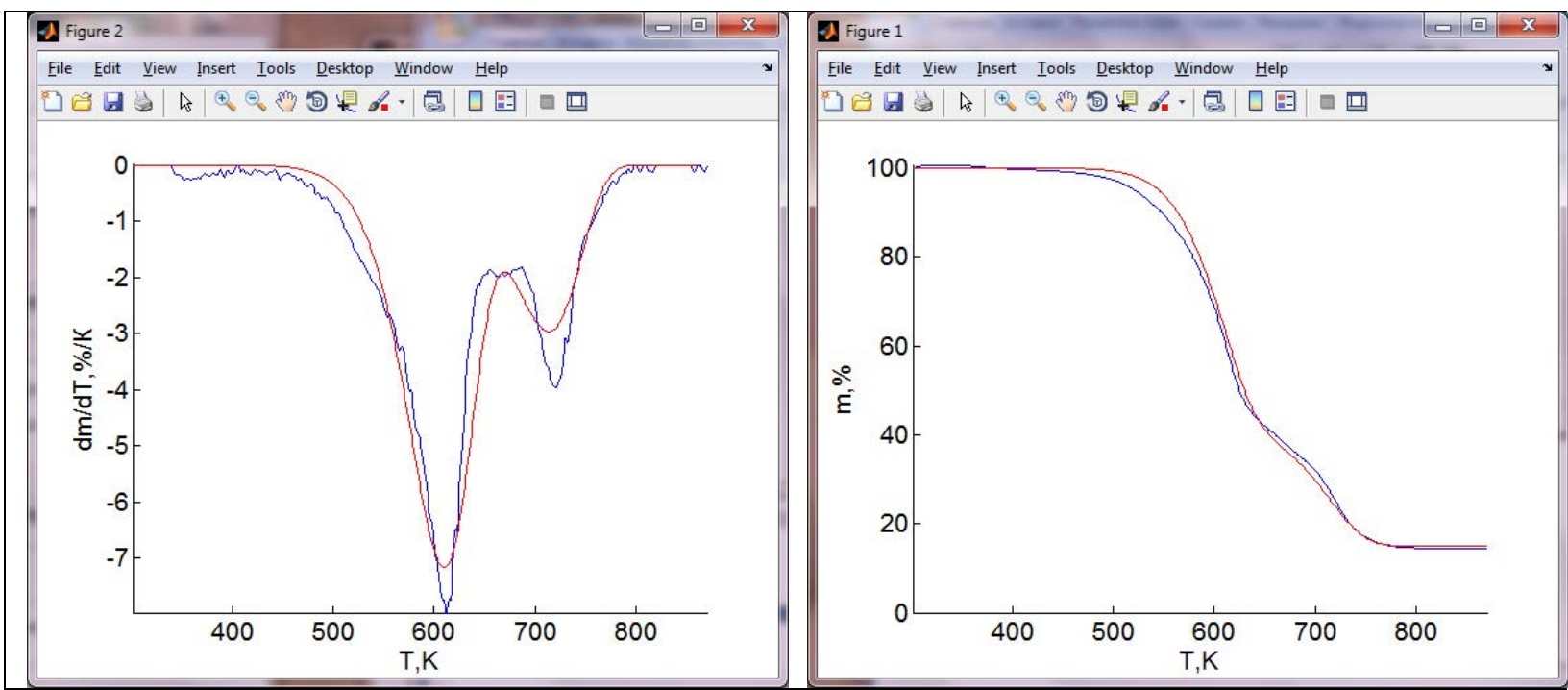

Рисунок 2 - Экспериментальные данные и подбор термокинетических постоянныхдля экспериментальных данных с 10\% кислорода. 


\section{5. Выводы}

Согласно полученным результатам выбранная математическая модель пиролиза позволяет точно предсказывать скорость потери массы вещества при пиролизе в окислительной среде, если термокинетические постоянные найдены точно. Разработанный алгоритм позволяет обеспечить точность подбора энергии активации и предэкспоненциального множителя для двухстадийного пиролиза и минимизировать отклонения теоретической кривой от экспериментальных данных.

\section{Список литературы:}

1. Волков Е. А. Глава 1. Приближение функций многочленами. § 11. Сплайны // Численные методы. - Учеб. пособие для вузов. - 2-е изд., испр.. - М.: Наука, 1987. - С. 63-68. - 248 с.

2. Miller RS and Bellan J, Analysis of Reaction Products and Conversion Time in the Pyrolysis of Cellulose and Wood Particles., Pasadena, 1996.

3. Matala A., Lautenberger C., and Hostikka S. Generalized direct method for pyrolysis kinetic parameter estimation and comparison to existing methods // Journal of Fire Sciences. 2012. Vol. 30. No. 4. pp. 339-356. 